\title{
Analysis and Improvement of Attitude Output Accuracy in Rotation Inertial Navigation System
}

\author{
Kui Li, ${ }^{1,2}$ Pengyu Gao, ${ }^{2}$ Lei Wang, ${ }^{2}$ and Qian Zhang ${ }^{2}$ \\ ${ }^{1}$ School of Electronic and Information Engineering, Beihang University, Beijing 100191, China \\ ${ }^{2}$ School of Instrument Science and Opto-Electronics Engineering, Beihang University, Beijing 100191, China \\ Correspondence should be addressed to Kui Li; xflikui@126.com
}

Received 7 May 2015; Accepted 2 July 2015

Academic Editor: Jian Guo Zhou

Copyright (c) $2015 \mathrm{Kui} \mathrm{Li} \mathrm{et} \mathrm{al.} \mathrm{This} \mathrm{is} \mathrm{an} \mathrm{open} \mathrm{access} \mathrm{article} \mathrm{distributed} \mathrm{under} \mathrm{the} \mathrm{Creative} \mathrm{Commons} \mathrm{Attribution} \mathrm{License,} \mathrm{which}$ permits unrestricted use, distribution, and reproduction in any medium, provided the original work is properly cited.

\begin{abstract}
Inertial navigation system (INS) measures vehicle's angular rate and acceleration by orthogonally mounted tri-axis gyroscopes and accelerometers and then calculates the vehicle's real-time attitude, velocity, and position. Gyroscope drifts and accelerometer biases are the key factors that affect the navigation accuracy. Theoretical analysis and experimental results show that the influence of gyroscope drifts and accelerometer biases can be restrained greatly in rotation INS (RINS) by driving the inertial measurement unit (IMU) rotating regularly, thus improving navigation accuracy significantly. High accuracy in position and velocity should be matched with that in attitude theoretically since INS is based on dead reckoning. However, the marine and vehicle experiments show that short-term attitude output accuracy of RINS is even worse compared with that of nonrotation INS. The loss of attitude accuracy has serious impacts on many task systems where high attitude accuracy is required. This paper researched the principle of attitude output accuracy loss in RINS and then proposed a new attitude output accuracy improvement algorithm for RINS. Experiment results show that the proposed attitude compensation method can improve short-term pitch and roll output accuracy from 20 30 arc seconds to less than 5 arc seconds and azimuth output accuracy improved from $2 \sim 3$ arc minutes to less than 0.5 arc minutes in RINS.
\end{abstract}

\section{Introduction}

Inertial navigation systems (INS) employ inertial sensors (gyroscopes and accelerometers) to establish an inertial platform for measuring vehicle's angular rate and acceleration and then calculate vehicle's attitude, velocity, and position based on dead reckoning principle. Traditional inertial platforms are usually constructed physically by three gimbals at least to isolate vehicle's angular motion. With the development of technology, inertial platform INS is replaced by strapdown INS gradually, in which the inertial sensors are fixed along body axes and the physical platform is constructed mathematically by the attitude transformation matrix. Strapdown configurations could reduce system cost and increase system reliability greatly compared with physical platform INS, while, in both physical platform system and strapdown system, position errors accumulate with time because of gyroscope drifts and accelerometer biases. However, if the inertial measurement unit (IMU) is forced to rotate along given axes regularly, gyroscopes drifts and accelerometer bias errors can be modulated from constant to periodically varying components, thus attenuating system errors prominently.

Research on rotation INS (RINS) has been carried out in the past. Pioneering research was presented by Geller in 1968, which described an inertial platform INS with continuous platform rotation relative to the local geodetic frame [1]. Laser gyroscopes applied to RINS emerged from the 1980s $[2,3]$. A laser gyroscope RINS with discontinuous rotation was proposed by San Giovanni Jr. and Levinson in 1981 [4]. In 2004, Yang and Miao analyzed single-axis continuous rotation INS based on fiber-optic gyroscopes [5]. In 2007, Ishibashi et al. proved that position accuracy would acquire great improvement by driving INS rotate continuously on a turntable during alignment and navigation $[6,7]$. Dualaxis RINS was brought out to solve the defects that vertical axis inertial sensors errors cannot be restrained in singleaxis RINS $[8,9]$. In dual-axis RINS, whether it is based on continuous rotation scheme [10] or consequential rotation 


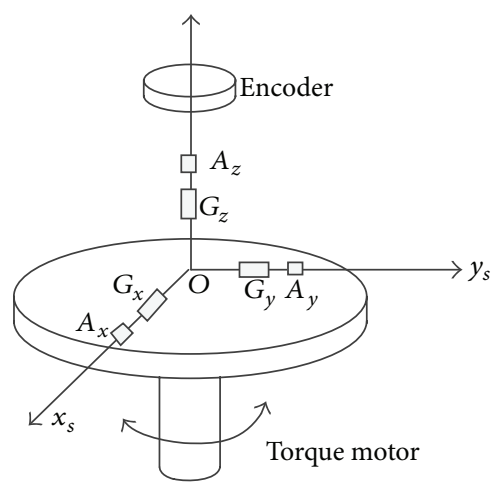

FIGURE 1: System structure.

scheme [11], inertial sensor errors along three directions can be restricted simultaneously. Besides, the USA has started the IFOG strategic nuclear submarine plan and expected to manufacture the first tri-axis RINS system in 2010 [12]. The tri-axis RINS system is designed with three-axis continuous rotation scheme [13] and can compensate the scale factor error of sensors, installation axis instability, gyroscope drifts, and so forth. These papers demonstrate that RINS has been widely researched and has huge prospect for improving INS navigation accuracy. However, most research on RINS aims at velocity and position accuracy improvement, which is also verified by RINS experimental results in this paper. But it should be noticed that RINS experimental results also show that short-term attitude output accuracy of RINS gets even worse compared with that of nonrotation INS. In some airborne, shipborne, or land vehicles applications, high accuracy attitude information output is more crucial for some task systems and loss of attitude accuracy will lead to serious consequences. Since INS is based on dead reckoning principle, high accuracy in position and velocity should be matched with attitude accuracy. This paper researched the reasons that caused the loss of RINS attitude output accuracy and then presented the corresponding attitude accuracy improvement algorithm. Section 2 described the RINS system configuration and analyzed errors restraint principle and preliminary experiment results. Section 3 presented RINS attitude update algorithm and researched the possible reasons of attitude output accuracy loss. Section 4 proposed the designed RINS attitude compensation algorithm and presented the experimental verification results. Conclusions were drawn in Section 5.

\section{RINS Error Restraint Analysis and Experiment Verification}

2.1. RINS Error Restraint Analysis. The system structure of the experimental RINS is shown in Figure 1. The IMU mainly includes three Ring Laser Gyroscopes (RLG) and three quartz accelerometers. The torque motor drives IMU to rotate along the vertical body axis bidirectionally and an angular encoder is fixed to provide rotation angle relative to the body frame.
TABLE 1: Main components' specification of RINS.

\begin{tabular}{lc}
\hline Gyro drifts & $0.008^{\circ} / \mathrm{h}$ \\
\hline Accelerometers biases & $50 \mu \mathrm{g}$ \\
\hline Encoder resolution & $1.08^{\prime} / \mathrm{pulse}$ \\
\hline Rotation angular rate & $0.686^{\circ} / \mathrm{s}$ \\
\hline
\end{tabular}

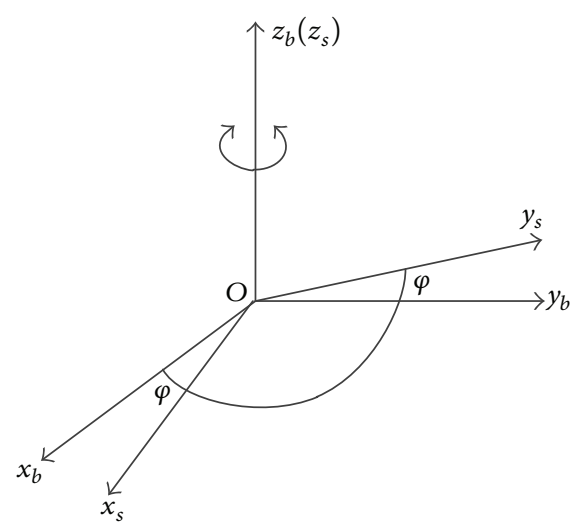

FIGURE 2: Frame definition.

Table 1 shows the main components' specification of the experimental RINS.

The proposed encoder provides digital output of 20000 pulses per $360^{\circ}$ rotation, and the resolution is $1.08^{\prime} /$ pulse. The experimental RINS takes the scheme of bidirectionally continuous rotation, and the rotation period is $1050 \mathrm{~s}$. Considering IMU rotation, the experimental RINS involves a new frame named inertial sensing frame ( $s$ frame, $O-x_{s} y_{s} z_{s}$ ). As is shown in Figure 2, $s$ frame refers to the rotation frame, which varies with the change of inertial sensors' pointing direction in real time. The body frame ( $b$ frame, $O-x_{b} y_{b} z_{b}$, right-forward-upward) is defined the same as $s$ frame when rotation angle $\varphi$ is zero.

The errors restraint principle of RINS can be explained by the following analysis. At time $t$, the angular rate and specific force output of the gyroscopes and accelerometers are

$$
\begin{aligned}
\omega_{i s}^{s} & =C_{b}^{s} \omega_{i b}^{b}+\left[\begin{array}{lll}
\varepsilon_{x} & \varepsilon_{y} & \varepsilon_{z}
\end{array}\right]^{T}+\omega_{b s}^{s}, \\
f_{i s}^{s} & =C_{b}^{s} f_{i b}^{b}+\left[\begin{array}{lll}
\nabla_{x} & \nabla_{y} & \nabla_{z}
\end{array}\right]^{T},
\end{aligned}
$$

where $\omega_{i b}^{b}$ and $f_{i b}^{b}$ denote ideal gyroscopes and accelerometers outputs along $b$ frame. $C_{s}^{b}$ denotes the coordinatetransformation matrix between $b$ frame and $s$ frame. $\varepsilon_{x}, \varepsilon_{y}, \varepsilon_{z}$ are gyroscope drifts and $\nabla_{x}, \nabla_{y}, \nabla_{z}$ are accelerometer biases. $\omega_{b s}^{s}=\left[\begin{array}{lll}0 & 0 & \omega\end{array}\right]^{T}$ represents rotation rate projected on $s$ frame. Afterwards, the angular rate and the specific force in body frame can be obtained by the following equations:

$$
\begin{aligned}
\widetilde{\omega}_{i b}^{b} & =C_{s}^{b} \omega_{i s}^{s}+\omega_{s b}^{b}, \\
\widetilde{f}_{i b}^{b} & =C_{s}^{b} f_{i s}^{s},
\end{aligned}
$$


where $\widetilde{\omega}_{i b}^{b}$ and $\widetilde{f}_{i b}^{b}$ represent gyroscope and accelerometer actual outputs and $\omega_{s b}^{b}=-C_{s}^{b} \omega_{b s}^{s}=\left[\begin{array}{lll}0 & 0 & -\omega\end{array}\right]^{T}$. Then (3) can be obtained by substituting (1) into (2) as follows:

$$
\begin{aligned}
& {\left[\begin{array}{c}
\widetilde{\omega}_{i b x}^{b} \\
\widetilde{\omega}_{i b y}^{b} \\
\widetilde{\omega}_{i b z}^{b}
\end{array}\right]=\omega_{i b}^{b}+\left[\begin{array}{c}
\varepsilon_{x} \cos \varphi-\varepsilon_{y} \sin \varphi \\
\varepsilon_{x} \sin \varphi+\varepsilon_{y} \cos \varphi \\
\varepsilon_{z}
\end{array}\right],} \\
& {\left[\begin{array}{c}
\widetilde{f}_{i b x}^{b} \\
\widetilde{f}_{i b y}^{b} \\
\widetilde{f}_{i b z}^{b}
\end{array}\right]=f_{i b}^{b}+\left[\begin{array}{c}
\nabla_{x} \cos \varphi-\nabla_{y} \sin \varphi \\
\nabla_{x} \sin \varphi+\nabla_{y} \cos \varphi \\
\nabla_{z}
\end{array}\right],}
\end{aligned}
$$

where $\varphi$ denotes the rotation angle between $b$ frame and $s$ frame provided by the encoder. If the rotation rate of IMU is ideal, the rotation angle $\varphi$ equals $\omega t$ during forward rotation and $-\omega t$ during reverse rotation.

In traditional strapdown INS, constant inertial sensor errors (gyro drifts and accelerometer biases) in $b$ frame are the main contributor to INS positioning error, while in RINS inertial sensor errors turn into periodical components. Then after dead reckoning calculation, attitude errors, horizontal velocity errors, and horizontal position errors caused by gyroscope drifts and accelerometer biases can be modulated, so navigation errors in RINS are no longer accumulated with time and navigation accuracy could achieve great improvement.

2.2. RINS Experimental Verification Results. In order to compare the error restraint effect brought about by IMU rotation, the experimental RINS can be transformed into traditional strapdown INS by locking the torque motor rotation axis while navigation algorithm remains unchanged. Under the same working condition, comparative experiments are conducted in both rotation and strapdown mode which last $4200 \mathrm{~s}$ ( 4 bidirectional rotation periods). The proposed RINS prototype and associated experimental equipment and their connections are shown in Figure 3. The RINS prototype is mounted on a dual-axis turntable; the experimental data are collected by a laptop.

Experimental results are given as follows: Figure 4 shows horizontal position errors, Figure 5 shows horizontal velocity errors, and Figure 6 shows attitude errors. The dashed curve represents strapdown results, and the solid curve represents rotation results.

In Figures 4 and 5, $\mathrm{E}$ is short for east and $\mathrm{N}$ is short for north. The maximum of horizontal positioning error in the strapdown mode is $1500 \mathrm{~m}$ during experiment while maximum positioning error is only $160 \mathrm{~m}$ in rotation mode, which means that positioning accuracy is improved nearly 10 times in RINS. The maximum of horizontal velocity error in the strapdown mode is $1.5 \mathrm{~m} / \mathrm{s}$ while maximum velocity error in rotation mode is less than $0.3 \mathrm{~m} / \mathrm{s}$. It means velocity accuracy is improved about 5 times. Thus, it can be seen that, by driving IMU rotation bidirectionally, horizontal position and velocity accuracy are improved obviously in RINS.

The attitude outputs during comparative experiment are given in Figure 6. It can be seen that, in strapdown mode,

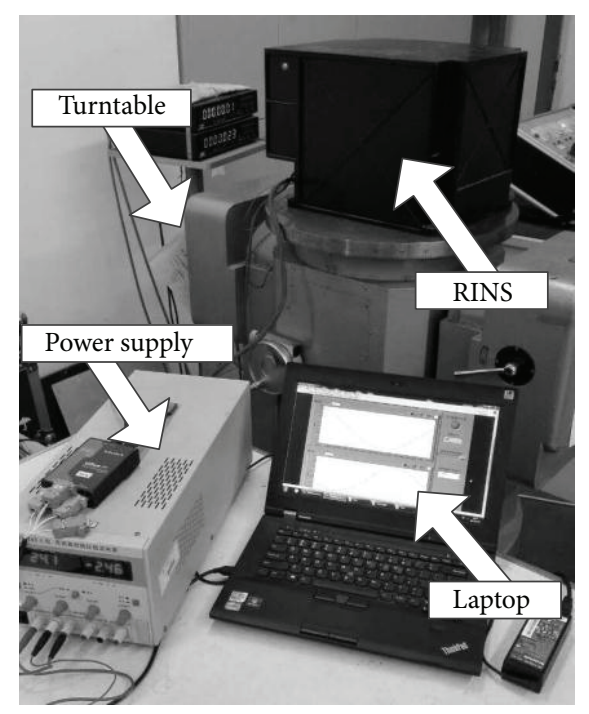

FIGURE 3: RINS and associated experimental equipment.

attitude error changes slowly during experiment and it shows good stability in short term (several minutes). The relatively long-term periodical varying components in pitch and roll errors are Schuler oscillations ( $84.4 \mathrm{~min}$ period). However, in rotation mode, attitude errors become more complicated. Pitch and roll errors are stable on the general trend during the whole 70-minute experiment. The mean value of attitude errors in every rotation period is nearly the same. It is hard to see Schuler oscillations in the error curve because most of horizontal inertial sensor errors have been attenuated by IMU rotation; thus Schuler oscillations amplitude is small. However, the short-term periodical fluctuation arises in attitude outputs with oscillations amplitude of 20 30 arc seconds in pitch and roll errors and 2 3 arc minutes in azimuth errors. Compared to strapdown mode, position and velocity accuracy in RINS increased greatly but attitude errors fail to reach the same accuracy in short term (even get worse), let alone improvement by IMU rotation. But for some airborne, shipborne, or land vehicle task systems, not only is high accuracy positioning and velocity reference needed, but also high accuracy attitude reference is important. Error fluctuation of 20 arc seconds in pitch and roll output and error fluctuation of 2 arc minutes in azimuth output will have seriously negative effect on their performance. However, according to theoretical analysis mentioned in Section 2, high accuracy in position and velocity should be matched with high attitude accuracy since INS is based on dead reckoning principle. Therefore, it is very necessary to analyze causes of the attitude output fluctuation errors and to find solutions to improve short-term attitude accuracy in RINS.

\section{Analysis of Attitude Output Accuracy in RINS}

3.1. Attitude Update Algorithm in RINS. The body's attitude in INS can be described by transformation matrix from $b$ frame to $n$ frame (navigation frame) through attitude matrix $C_{b}^{n}$. In 

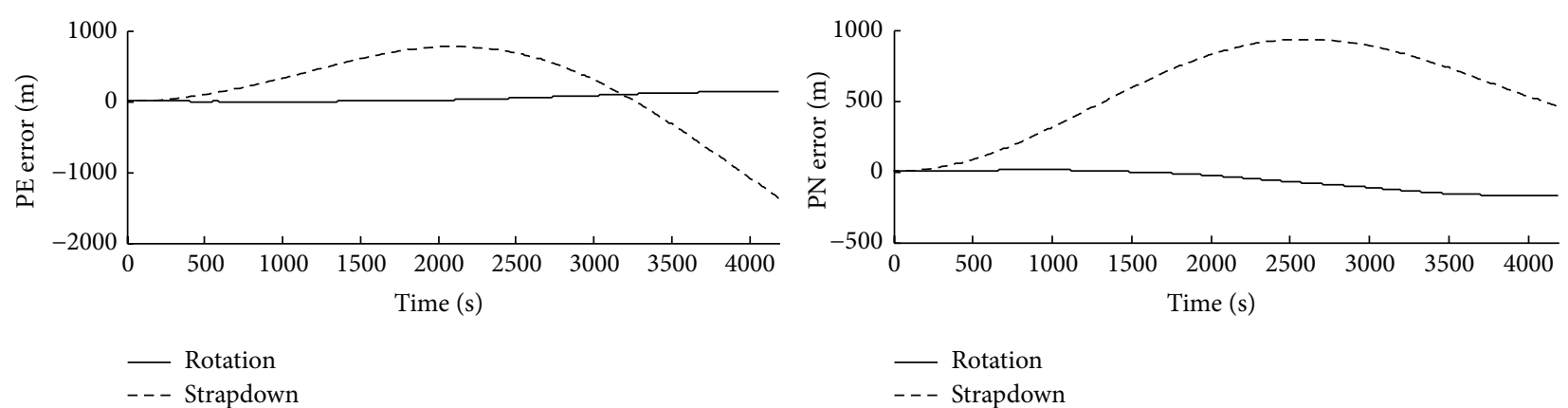

FIGURE 4: PE and PN errors comparison.
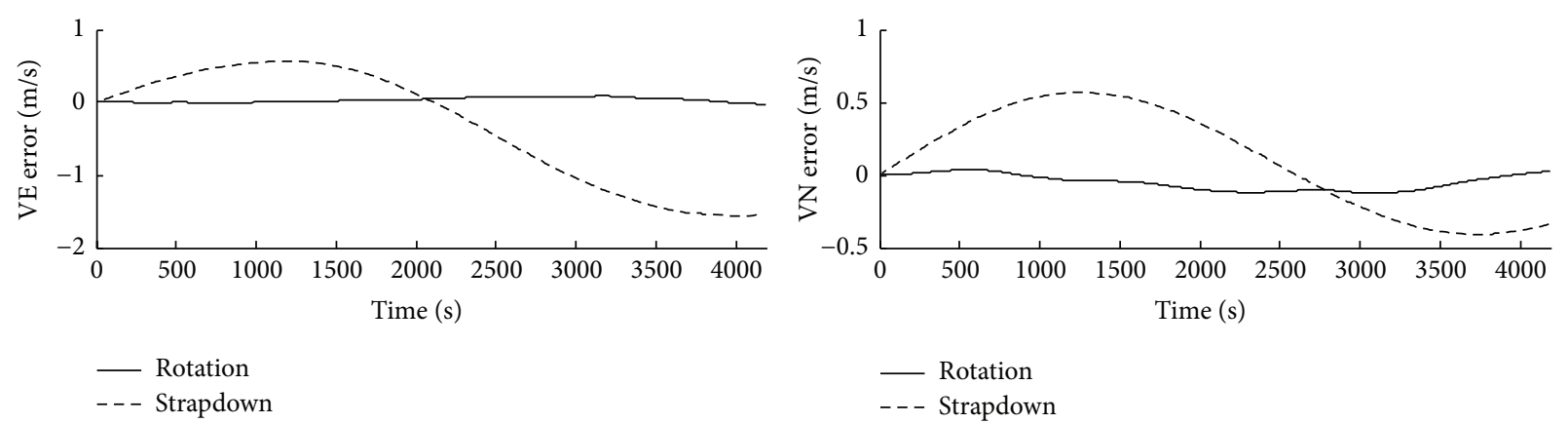

Figure 5: VE and VN errors comparison.
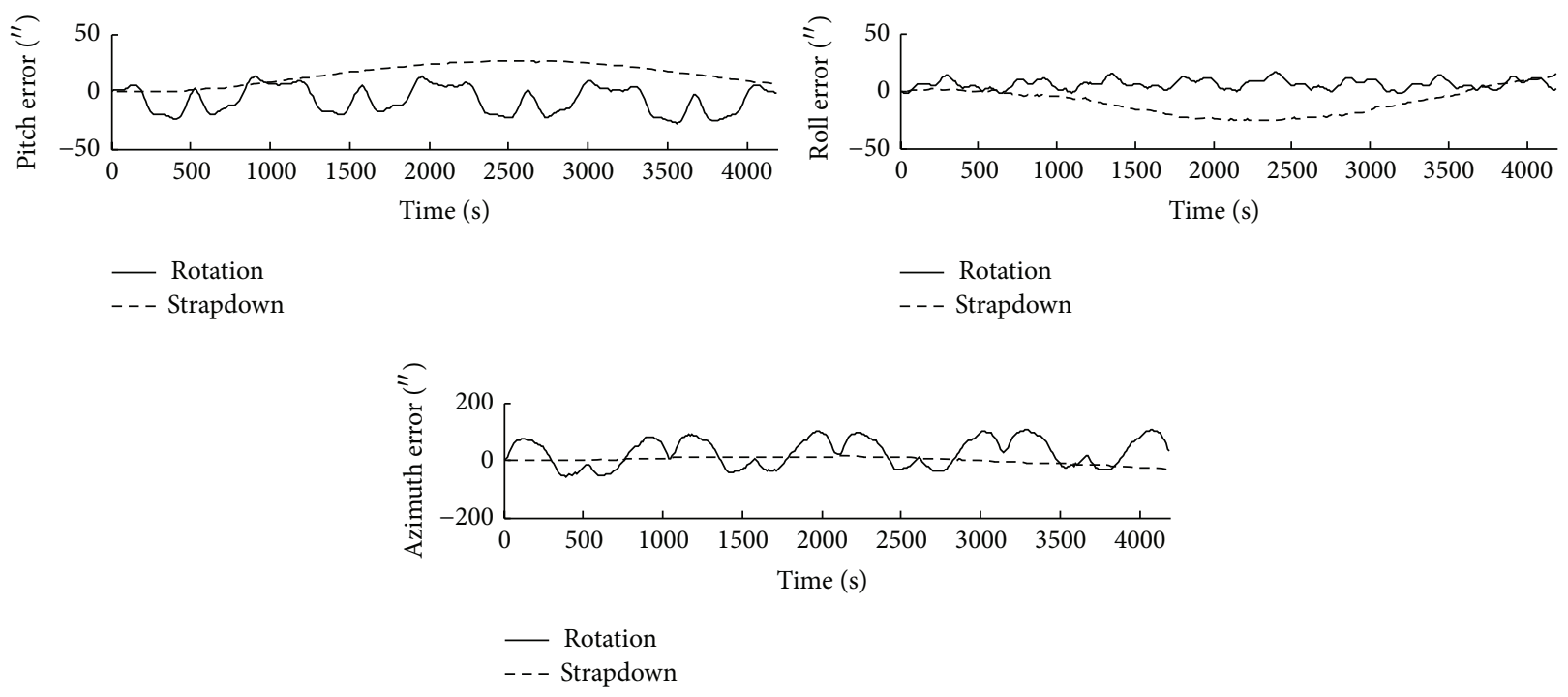

FIGURE 6: Strapdown and rotation INS attitude errors comparison.

strapdown INS configuration, $C_{b}^{n}$ is calculated directly from rotation vector by quaternion attitude updating algorithm. However, in RINS configuration, attitude matrix $C_{s}^{n}$ should be updated beforehand (the update process of matrix $C_{s}^{n}$ is the same as that of $C_{b}^{n}$ in strapdown INS); then $C_{b}^{n}$ can be acquired according to the following equation:

$$
C_{b}^{n}=C_{s}^{n} C_{b}^{s}
$$

The matrix $C_{b}^{s}$ is the transformation matrix from $b$ frame to $s$ frame and is given as follows:

$$
C_{b}^{s}=\left[\begin{array}{ccc}
\cos \varphi & \sin \varphi & 0 \\
-\sin \varphi & \cos \varphi & 0 \\
0 & 0 & 1
\end{array}\right],
$$

where $\varphi$ is the rotation angle provided by encoder. 
If all the updating processes above are ideal, attitude output in RINS should present high accuracy and should not contain short-term error fluctuation. As a matter of fact, in RINS, updating matrix $C_{s}^{n}$ is calculated from rotation vector by quaternion attitude updating algorithm, which is the same as $C_{b}^{n}$ in strapdown INS; thus it is accurate enough. Consequently, the most possible cause of RINS short-term attitude output accuracy loss is transformation process from attitude matrix $C_{s}^{n}$ to $C_{b}^{n}$, that is to say, matrix $C_{b}^{s}$. In actual RINS, the encoder output errors, installation eccentricity of encoder, and noncoaxial rotation of IMU can cause rotation angle $\varphi$ containing periodical output angle errors [14], which lead to azimuth output fluctuation in RINS. Besides, if the axis $\mathrm{O}-z_{s}$ does not coincide with the rotation axis $\mathrm{O}-z_{b}$ all the time, the rotation angular rate will have projection along horizontal axes $O-x_{s}$ and $O-y_{s}$. Thus, undesirable influences will be attached to the transformation matrix $C_{b}^{s}$, which will result in error fluctuation on pitch and roll output. The detailed analysis will be given as follows.

3.2. Analysis of Azimuth Output Error in RINS. Equation (5) indicates that the rotation angle $\varphi$ will have effect on the accuracy of matrix $C_{b}^{s}$. Therefore, it is necessary to analyze the rotation angle provided by the encoder first. In order to obtain the encoder angle errors, the angle integrated by output angular rate of gyroscope $Z$ is used. However, output of gyroscope $Z$ contains not only rotation angular rate $\omega$, but also gyroscope drift $\varepsilon_{z}$ and the upward component of earth rotation angular rate $\omega_{i e} \sin L$. The sign of IMU rotation rate $\omega$ is opposite during forward and reverse rotation. Then the gyroscope outputs are given as

$$
\begin{aligned}
& \omega_{z f}=\omega+\omega_{i e} \sin L+\varepsilon_{z}, \\
& \omega_{z r}=-\omega+\omega_{i e} \sin L+\varepsilon_{z},
\end{aligned}
$$

where subscript $f$ denotes forward rotation and $r$ denotes reverse rotation. If we define $\omega^{\prime}$ as $\omega_{i e} \sin L+\varepsilon_{z}=\left(\omega_{z f}+\right.$ $\left.\omega_{z r}\right) / 2$, then the angle integrated by output angular rate of gyroscope $Z$ during IMU rotation can be calculated according to (7), which will be a reference for encoder angle output. Consider

$$
\begin{aligned}
\varphi_{z f} & =\int\left(\omega_{z f}-\omega^{\prime}\right) d t, \\
\varphi_{z r} & =\int\left(\omega_{z r}-\omega^{\prime}\right) d t .
\end{aligned}
$$

Figure 7 shows actual experimental data of encoder angle outputs and Figure 8 is acquired when subtracting encoder angle from the integrated angle of gyroscope $Z$. It can be seen from the two figures that encoder angle error (define as $\delta \varphi$ ) presents obvious repeatability during 4 bidirectional rotation periods, and the fluctuations are axial symmetry about forward and reverse rotation in a separate bidirectional period. $\delta \varphi$ presents periodical fluctuation with the same period as IMU rotation, and the fluctuation amplitude is $1 \sim$ 2 arc minutes. It presents the same property as azimuth error in Figure 6 and can be compensated; afterwards, short-term azimuth output accuracy would be greatly improved.

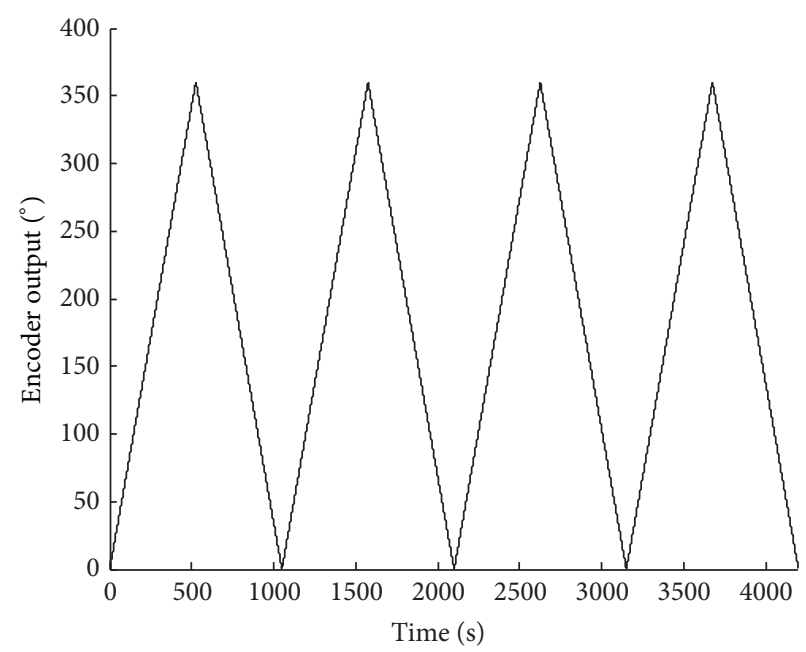

Figure 7: Encoder angular output.

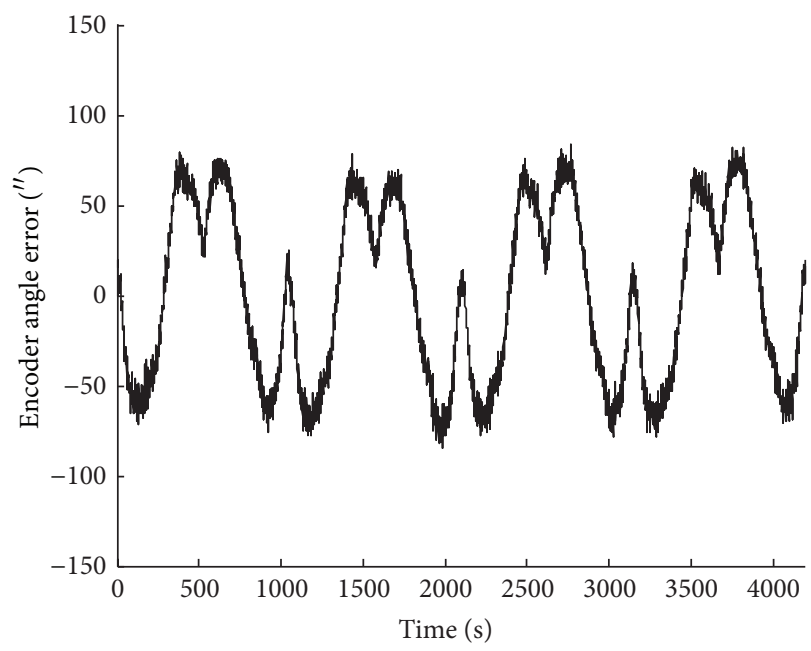

Figure 8: Encoder angular error.

3.3. Analysis of Pitch and Roll Output Error in RINS. The expression of matrix $C_{b}^{s}$ in (5) is obtained based on ideal rotation; namely, the axes of $O-x_{s}$ and $O-y_{s}$ stay in a fixed plane during rotation. However, in fact, irregular rotation is hard to avoid and the rotation plane would change in different positions. The difference between actual rotation and ideal rotation should be analyzed in detail.

Figure 9 (left) describes IMU rotation under ideal condition, and in this case axes of $O-x_{s}$ and $O-y_{s}$ in $s$ frame remain unchanged in fixed plane and axis $O-z_{s}$ coincides with $O-z_{b}$ in body frame all the time. However, in actual condition, due to some reasons such as machining and assembling errors in rotation axis and defects in rotation bearings, the IMU rotates irregularly. Figure 9 (right) shows this irregular rotation and the rotation plane in $s$ frame no longer stays unchanged; the pointing of $O-z_{s}$ axis will change in space and axes of $O-x_{s}$ and $O-y_{s}$ will fluctuate around the ideal fixed plane.

If the rotation plane remains unchanged, the projection of acceleration of gravity on horizontal accelerometers will 

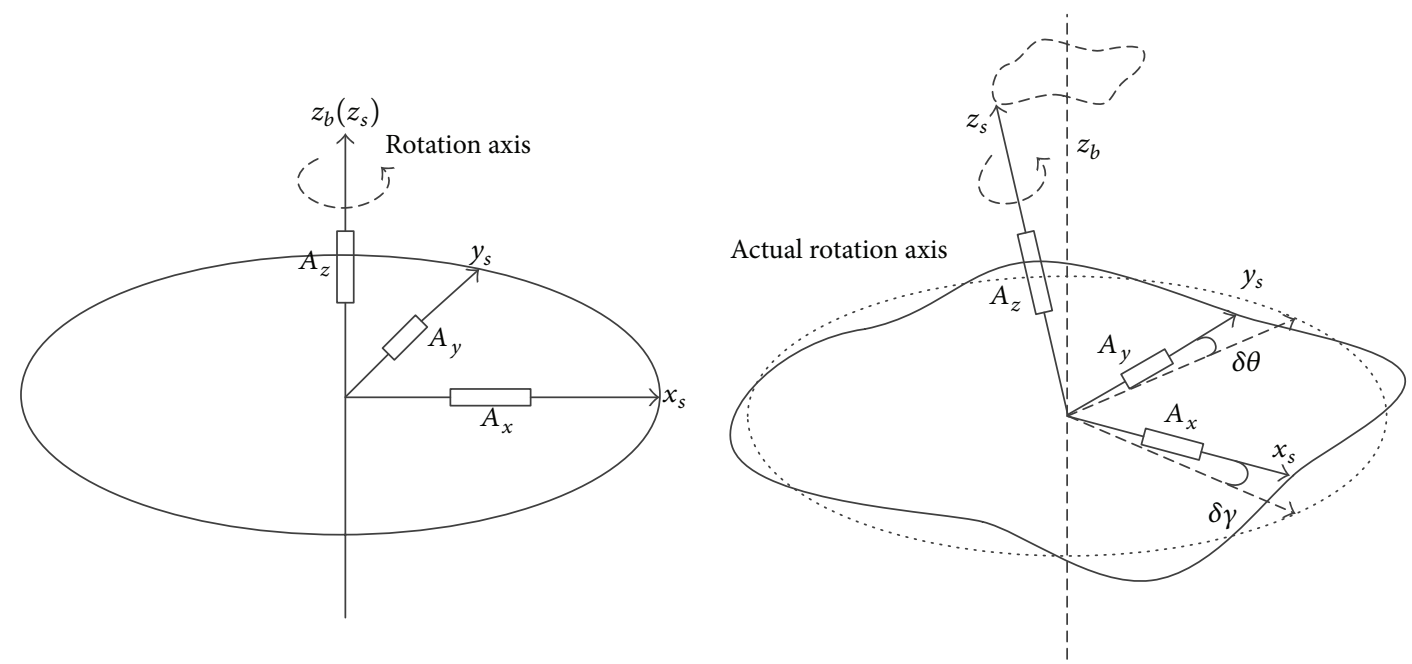

FIGURE 9: Comparison of ideal rotation and actual irregular rotation.
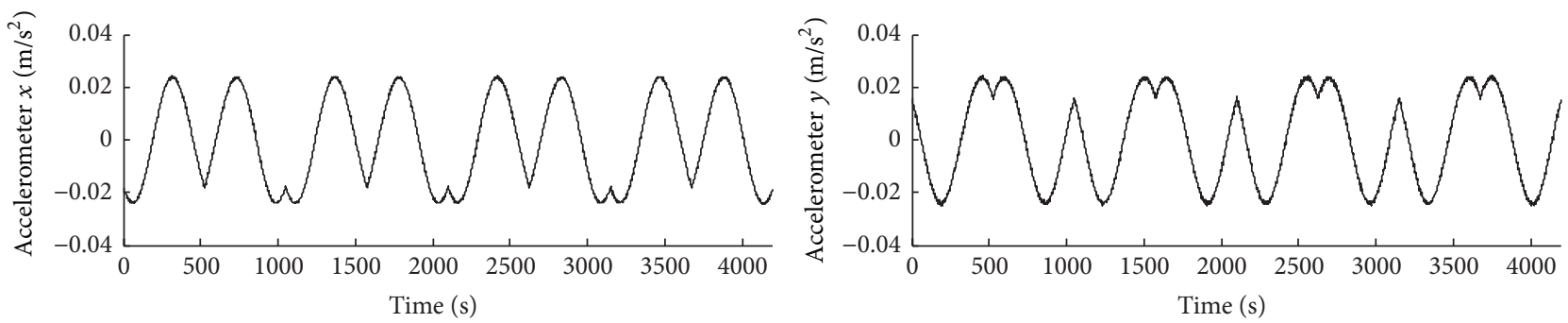

FIGURE 10: Initial outputs of horizontal accelerometers.
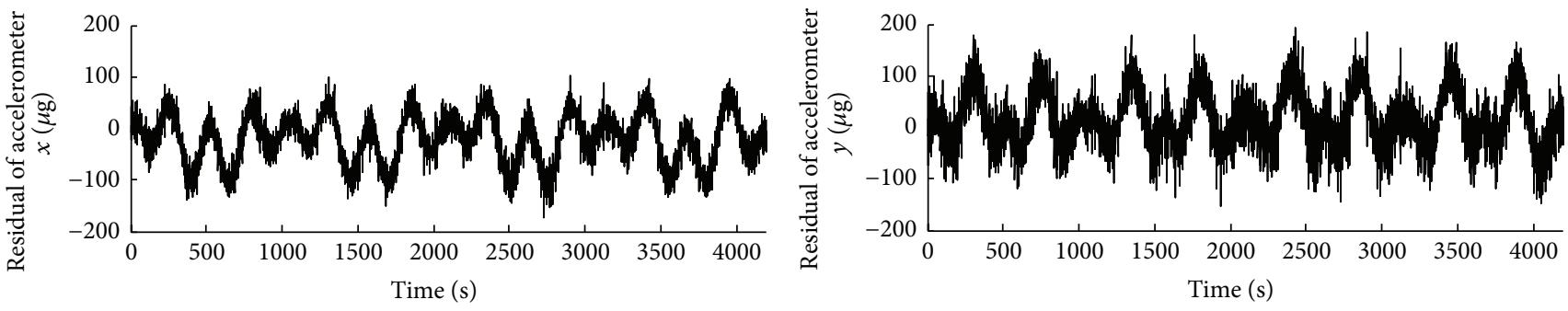

FIGURE 11: Residual components of horizontal accelerometers.

be standard sine or cosine form with the same period as IMU rotation, and the amplitude is determined by initial pitch and roll of the rotation plane. However, due to the fluctuation of rotation plane in actual situation, the projection of acceleration of gravity on horizontal accelerometers will bring about irregular variation, and the deviation between the rotation plane and the fixed plane can be represented by irregular variation in horizontal accelerometers. Figure 10 shows the original output of two horizontal accelerometers in actual experiments. In order to obtain the irregular rotation components, firstly, the projection of acceleration of gravity caused by pitch and roll should be deducted from the horizontal accelerometers according to

$$
\begin{aligned}
& a_{x}^{\prime}=a_{x}+g \gamma_{0} \cos \varphi-g \theta_{0} \sin \varphi, \\
& a_{y}^{\prime}=a_{y}-g \theta_{0} \cos \varphi-g \gamma_{0} \sin \varphi,
\end{aligned}
$$

where $\theta_{0}, \gamma_{0}$ are initial pitch and roll acquired by the alignment process in RINS.

Figure 11 shows the residual components of horizontal accelerometers during the experiment. Both horizontal accelerometers residual components mainly represent second harmonic frequency related to the rotation period and show good repeatability during 4 bidirectional rotation periods. In a single bidirectional period, the fluctuations on the horizontal accelerometers are also axial symmetry. The fluctuation amplitude is about $150 \mu \mathrm{g}$, which means the maximum deviation between the rotation plane and the fixed plane is about 30 arc seconds, and it coincides with the amplitude of pitch and roll output fluctuation in Figure 6.

The residuals of horizontal accelerometers can be transformed into deviation angles between the rotation plane and 


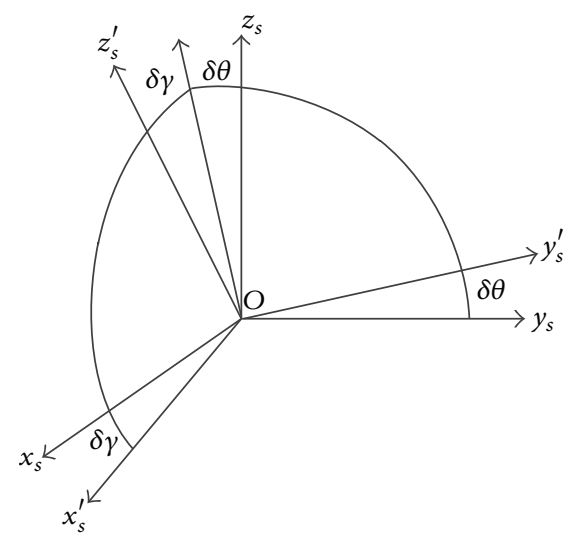

FIGURE 12: Transformation relation between $s$ frame and $s^{\prime}$ frame.

the fixed plane, which are defined as $\delta \theta, \delta \gamma$, as shown in Figure 9 (right), and are given as follows:

$$
\begin{aligned}
& \delta \theta=\frac{a_{y}^{\prime}}{g}, \\
& \delta \gamma=-\frac{a_{x}^{\prime}}{g} .
\end{aligned}
$$

To compensate influence caused by $\delta \theta, \delta \gamma$, another coordinate frame should be defined in the proposed RINS to distinguish actual irregular rotation from ideal rotation. The ideal rotation frame is named as $s^{\prime}$ frame, whose $O-z_{s}^{\prime}$ axis is coinciding with $O-z_{b}$ axis all the time. The previous $s$ frame is the actual rotation frame representing the instantaneous rotation frame, and it fluctuates around the ideal fixed plane. Taking into consideration irregular rotation, attitude matrix obtained by (6) should be described as $C_{s^{\prime}}^{n}$. The transformation matrix from $s$ frame to $s^{\prime}$ frame can be acquired by $\delta \theta$, $\delta \gamma$ and then the output attitude matrix $C_{b}^{n}$ can be updated by

$$
C_{b}^{n}=C_{s^{\prime}}^{n}{ }^{s^{\prime}} C_{b}^{s}
$$

Figure 12 shows the relationship between $s$ frame and $s^{\prime}$ frame. The transformation matrix $C_{s}^{s^{\prime}}$ can be obtained by two Euler angle rotations according to

$$
C_{s}^{s^{\prime}}=R_{y}(\delta \gamma) R_{x}(\delta \theta)
$$

where $R_{y}(\delta \gamma)=\left[\begin{array}{ccc}\cos \delta \gamma & 0 & -\sin \delta \gamma \\ 0 & 1 & 0 \\ \sin \delta \gamma & 0 & \cos \delta \gamma\end{array}\right]$, and $R_{x}(\delta \theta)=$ $\left[\begin{array}{ccc}1 & 0 & 0 \\ 0 & \cos \delta \theta & \sin \delta \theta \\ 0 & -\sin \delta \theta & \cos \delta \theta\end{array}\right]$

Since deviation angles $\delta \theta, \delta \gamma$ between $s$ frame and $s^{\prime}$ frame are small, the matrix $C_{s}^{s^{\prime}}$ can be simplified as follows:

$$
C_{s}^{s^{\prime}} \approx\left[\begin{array}{ccc}
1 & 0 & -\delta \gamma \\
0 & 1 & \delta \theta \\
\delta \gamma & -\delta \theta & 1
\end{array}\right]
$$

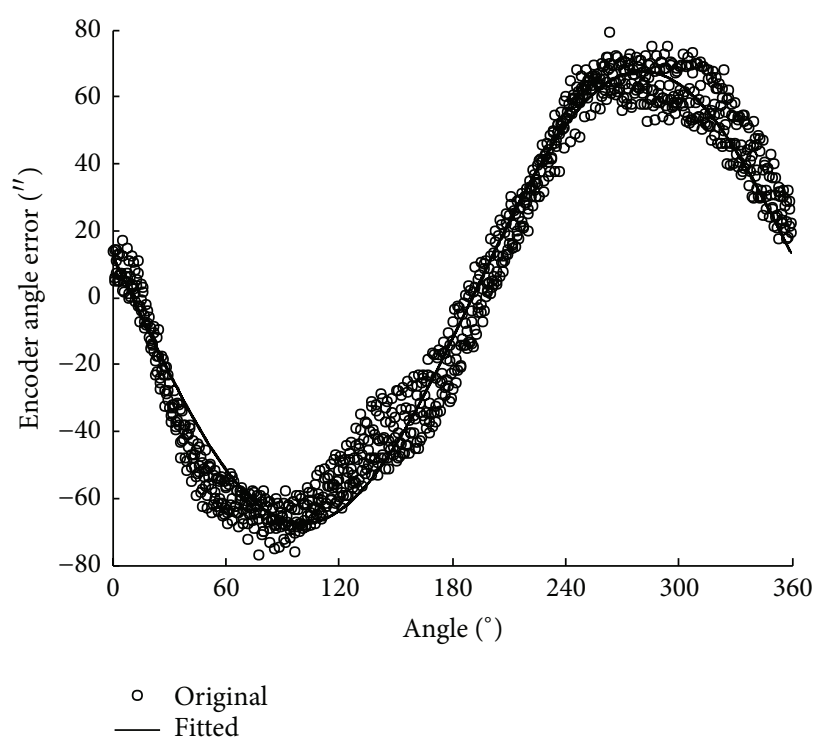

FIGURE 13: Relationship between encoder angle error and rotation angle.

\section{RINS Attitude Error Compensation and Experimental Verification}

According to the previous analysis, both encoder angle errors and axis irregular rotation can be corrected, and then RINS attitude error fluctuation can be mostly compensated, thus improving short-term attitude output accuracy.

4.1. Azimuth Output Correction Experimental Results in RINS. It can be seen in Figure 8 that $\delta \varphi$ is axial symmetry about forward and reverse rotation; thus it is closely related to encoder angle $\varphi$, and the relationship between $\delta \varphi$ and $\varphi$ is shown in Figure 13.

Figure 13 indicates that the fluctuation of $\delta \varphi$ presents fundamental frequency with respect to the rotation angle. Consequently, the error variation can be compensated through data fitting by the mathematical model given in

$$
\delta \varphi=k_{0}+k_{1} \sin \varphi+k_{2} \cos \varphi .
$$

Use $X$ to denote state variable, where $X=\left[\begin{array}{lll}k_{0} & k_{1} & k_{2}\end{array}\right]^{T}$, and then the coefficients $k_{0}, k_{1}, k_{2}$ can be acquired through least square fitting algorithm by the following measurements equation:

$$
Z=H X
$$

where $Z=\left[\begin{array}{llll}\delta \varphi_{1} & \delta \varphi_{2} & \cdots & \delta \varphi_{m}\end{array}\right]^{T}, H=\left[\begin{array}{ccc}1 & \sin \varphi_{1} & \cos \varphi_{1} \\ 1 & \sin \varphi_{2} & \cos \varphi_{2} \\ \cdots & \cdots & \cdots \\ 1 & \sin \varphi_{m} & \cos \varphi_{m}\end{array}\right]_{m \times 3}$, and $m$ denotes the total number of useful measurements.

Since the coefficients $k_{0}, k_{1}, k_{2}$ fitting results are acquired (in this RINS prototype, the coefficients fitting results are $k_{0}=0.032, k_{1}=-66.47$, and $\left.k_{2}=12.49\right)$, the encoder angle error in RINS could be corrected in real time by correcting coefficients $k_{0}, k_{1}, k_{2}$ according to 


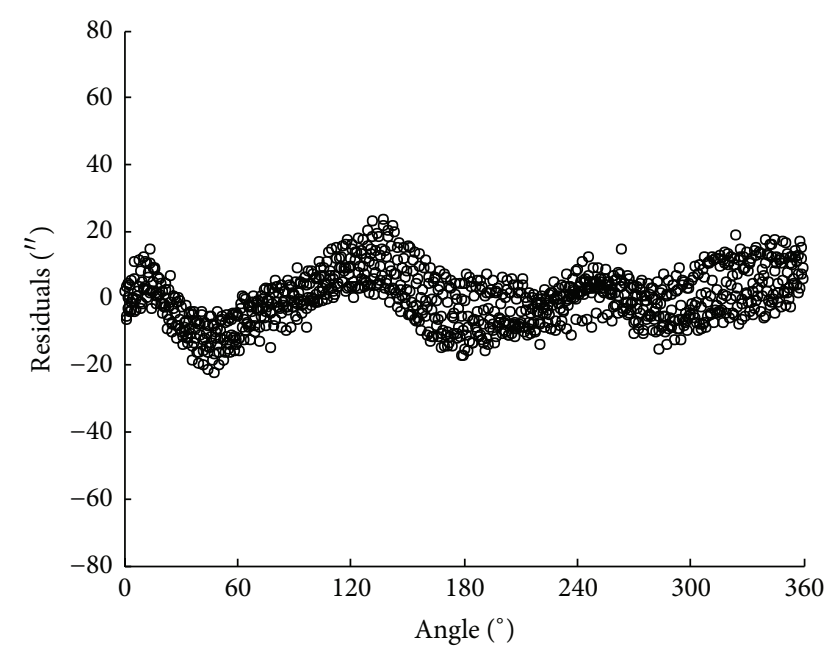

FIGURE 14: Fitting residuals of encoder angle errors.

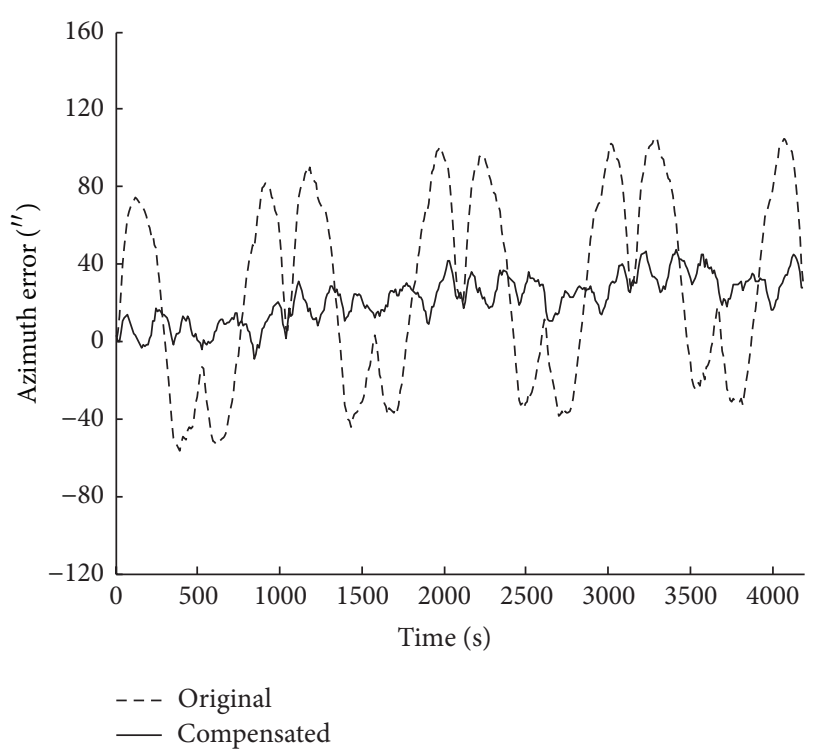

FIGURE 15: Comparison of original and compensated azimuth errors.

$$
\varphi_{C}=\varphi-\left(k_{0}+k_{1} \sin \varphi+k_{2} \cos \varphi\right),
$$

where $\varphi_{C}$ denotes the encoder angle to be compensated.

Figure 14 shows the fitting residuals of encoder angle errors and it is less than $20^{\prime \prime}$. Then the transformation matrix $C_{b}^{s}$ can be calculated by $\varphi_{C}$ instead of $\varphi$. The azimuth output correction results and comparisons are shown in Figure 15.

It can be seen from Figure 15 that, after azimuth output correction, the peak-to-peak azimuth errors decreased from 2 3 arc minutes to less than 0.5 arc minutes, which is improved nearly 5 times, and it means the short-term azimuth output accuracy achieves significantly improvement.

4.2. Pitch and Roll Compensation Results in RINS. The actual experimental data also show that, being similar to encoder angle errors, the derivation angles $\delta \theta, \delta \gamma$ acquired by residuals of horizontal accelerometers are axial symmetry with respect to encoder angle $\varphi$, which are shown in Figure 16, and
TABLE 2: Coefficients estimation results $\left({ }^{\prime \prime}\right)$.

\begin{tabular}{ccccc}
\hline & $a_{1}$ & $b_{1}$ & $a_{2}$ & $b_{2}$ \\
\hline$\delta \theta$ & -4.209 & -6.664 & 7.793 & 6.057 \\
$\delta \gamma$ & -6.414 & 4.108 & -0.127 & -9.708 \\
\hline
\end{tabular}

they can be used to correct and compensate pitch and roll output.

From Figure 16, it can be seen that the fluctuation of $\delta \theta$, $\delta \gamma$ mainly presents second harmonic frequency with respect to rotation period. Consequently, it can be modeled by (16), and the coefficients estimation results are shown in Table 2. Consider

$$
\begin{aligned}
& \delta \theta=a_{\theta 1} \sin \varphi+b_{\theta 1} \cos \varphi+a_{\theta 2} \sin 2 \varphi+b_{\theta 2} \cos 2 \varphi, \\
& \delta \gamma=a_{\gamma 1} \sin \varphi+b_{\gamma 1} \cos \varphi+a_{\gamma 2} \sin 2 \varphi+b_{\gamma 2} \cos 2 \varphi .
\end{aligned}
$$

Figure 17 shows the fitting residuals of $\delta \theta, \delta \gamma$ and they are less than $5^{\prime \prime}$. Then the transformation matrix $C_{b}^{s}$ can be calculated according to (10) and (11). Thus the pitch and roll output compensation results can be acquired, as shown in Figure 18.

Conclusions can be drawn from Figure 18 that, after pitch and roll output compensation, the peak-to-peak pitch and roll errors decreased from 20 30 arc seconds to less than 5 arc seconds, which is improved nearly 5 times. It is proved that the proposed output compensation algorithm for pitch and roll is valid, and the pitch and roll short-term output accuracy is improved obviously.

However, it is worth mentioning that the compensation algorithm here is not only suitable for single-axis rotation RINS. For dual-axis and tri-axis RINS, it is inevitable to have encoder installation eccentricity and other mentioned problems; meanwhile, the irregular rotation is hard to avoid as well; consequently, the analysis and attitude compensation and correction algorithm presented in this paper are also suitable for other types of RINS.

\section{Conclusion}

This paper researched the attitude output accuracy improvement in rotation RINS. Comparative experiment results indicate that velocity and position accuracy gains great improvement in rotation mode, while attitude accuracy is even worse than that in strapdown mode. The reasons that cause attitude output accuracy loss are analyzed, and then a new attitude output compensation algorithm for RINS is presented. The experimental results proved validity of the proposed attitude correction and compensation algorithm, with short-term pitch and roll output accuracy improved from 20 30 arc seconds to less than 5 arc seconds and azimuth output accuracy improved from $2 \sim 3$ arc minutes to less than 0.5 arc minutes.

According to dead reckoning principle, high accuracy in velocity and position should be matched with high attitude accuracy. The proposed compensation algorithm in this paper solved the problem of attitude accuracy loss of RINS in practical applications, which is significant for many task 

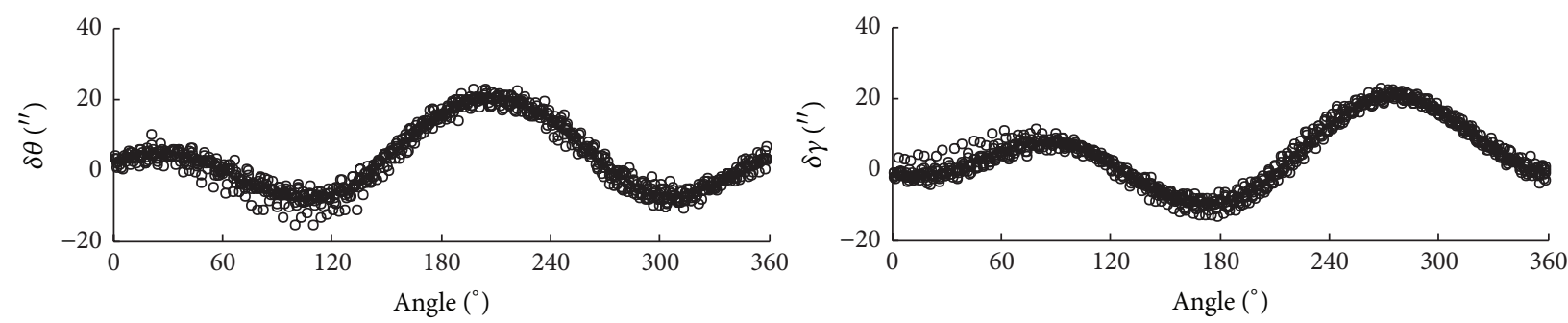

- Original

- Fitted

- Original

- Fitted

FIGURE 16: Relationships between $\delta \theta, \delta \gamma$, and rotation angle.
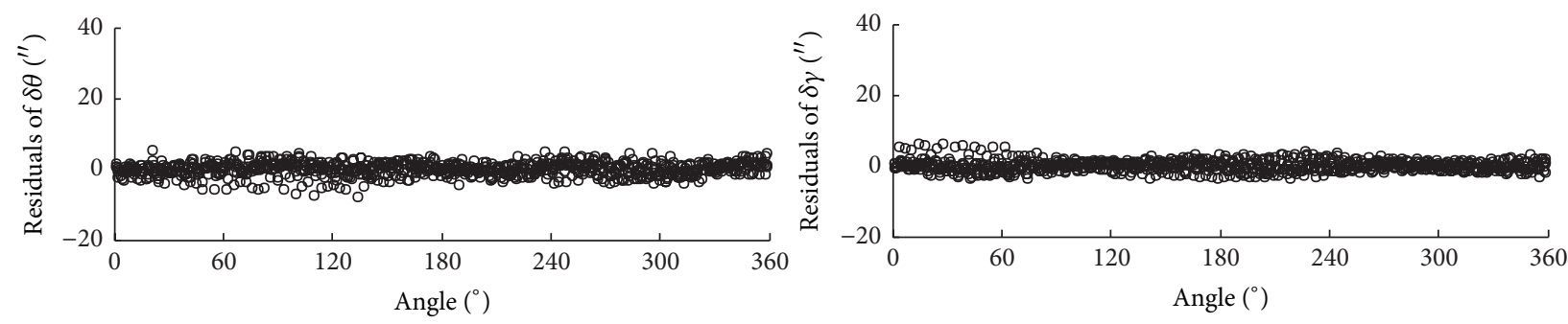

FIGURE 17: Fitting residuals of $\delta \theta, \delta \gamma$.
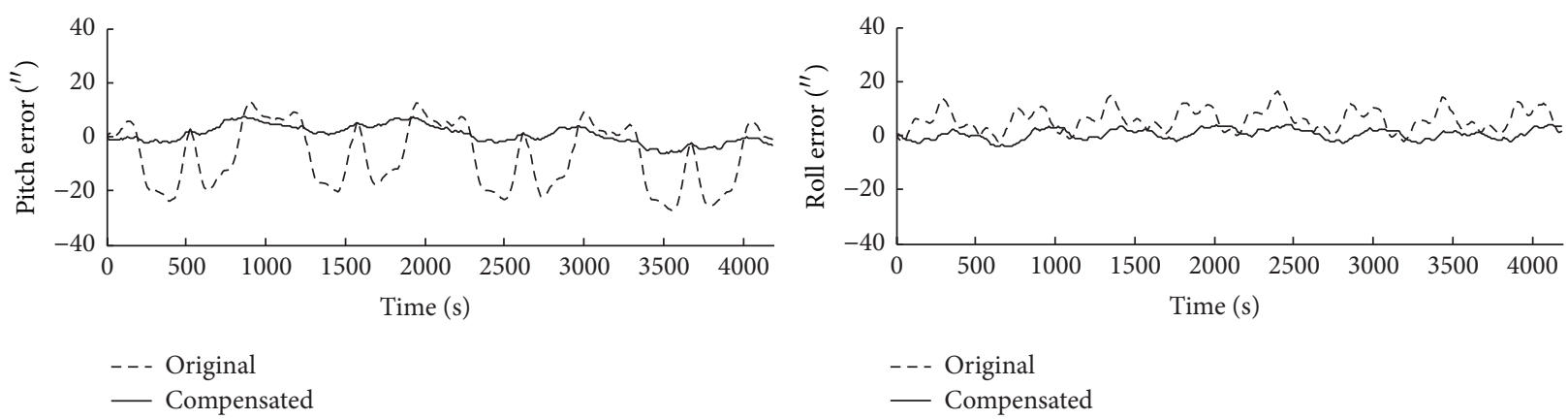

FIGURE 18: Comparison of original and compensated pitch and roll errors.

systems where attitude accuracy is urgently required. In addition, the proposed compensation algorithm is a general method; it is not only suitable for single-axis RINS, but it can also be used to improve short-term attitude output accuracy in dual-axis and tri-axis RINS.

\section{Conflict of Interests}

The authors declare that there is no conflict of interests regarding the publication of this paper.

\section{Acknowledgments}

This research is supported and funded by the National Natural Science Foundation of China (L142200032) and LongTerm Development Strategic Research of China Engineering Science and Technology (2014-zcq-01). The authors appreciate the support and fund.

\section{References}

[1] E. S. Geller, "Inertial system platform rotation," IEEE Transactions on Aerospace and Electronic Systems, vol. AES-4, no. 4, pp. 557-568, 1968.

[2] E. Levinson and R. Majure, "Accuracy enhancement techniques applied to the marine ring laser inertial navigator (MARLIN)," Navigation, vol. 34, no. 1, pp. 64-86, 1987.

[3] E. Levinson, J. ter Horst, and M. Willcocks, "The next generation marine inertial navigator is here now," in Proceedings of the Position Location and Navigation Symposium, pp. 121-127, IEEE, April 1994.

[4] C. San Giovanni Jr. and E. Levinson, "Performance of a ring laser strapdown marine gyrocompass," Navigation, Journal of the Institute of Navigation, vol. 28, no. 4, pp. 311-341, 1981.

[5] Y. Yang and L.-J. Miao, "Fiber-optic strapdown inertial system with sensing cluster continuous rotation," IEEE Transactions on Aerospace and Electronic Systems, vol. 40, no. 4, pp. 1173-1178, 2004 
[6] S. Ishibashi, S. Tsukioka, H. Yoshida et al., "Accuracy improvement of an inertial navigation system brought about by the rotational motion," in Proceedings of the OCEANS-Europe, pp. 1-5, IEEE, Aberdeen, Scotland, June 2007.

[7] S. Ishibashi, S. Tsukioka, T. Sawa et al., "The rotation control system to improve the accuracy of an inertial navigation system installed in an autonomous underwater vehicle," in Proceedings of the Symposium on Underwater Technology and Workshop on Scientific Use of Submarine Cables and Related Technologies, pp. 495-498, IEEE, Tokyo, Japan, April 2007.

[8] A. Li, G.-B. Chang, F.-J. Qin, and H.-W. Li, "Improved precision of strapdown inertial navigation system brought by dual-axis continuous rotation of inertial measurement unit," in Proceedings of the 2nd International Asia Conference on Informatics in Control, Automation and Robotics (CAR '10), vol. 1, pp. 284-287, March 2010.

[9] G. Chang, J. Xu, A. Li, and K. Cheng, "Error analysis and simulation of the dual-axis rotation-dwell autocompensating strapdown inertial navigation system," in Proceedings of the International Conference on Measuring Technology and Mechatronics Automation (ICMTMA '10), pp. 124-127, March 2010.

[10] Z. Deng, M. Sun, and B. Wang, "Error modulation scheme analysis of dual-axis rotating strap-down inertial navigation system based on FOG," in Proceedings of the 33rd Chinese Control Conference (CCC '14), pp. 692-696, Nanjing, China, July 2014.

[11] C. Jianhua, L. Mingyue, C. Daidai, C. Li, and S. Junyu, "Research of strapdown inertial navigation system monitor technique based on dual-axis consequential rotation," in Proceedings of the International Conference on Information and Automation (ICIA '11), pp. 203-208, June 2011.

[12] R. B. Morrow Jr. and D. W. Heckman, "High precision IFOG insertion into the strategic submarine navigation system," in Proceedings of the IEEE Position Location and Navigation Symposium, pp. 332-338, IEEE, Palm Springs, Calif, USA, April 1998.

[13] D. W. Heckman and L. M. Baretela, "Improved affordability of high precision submarine inertial navigation by insertion of rapidly developing fiber optic gyro technology," in Proceedings of the IEEE Position, Location and and Navigation Symposium, pp. 404-410, March 2000.

[14] S. Qin, Z. Huang, and X. Wang, "Optical angular encoder installation error measurement and calibration by ring laser gyroscope," IEEE Transactions on Instrumentation and Measurement, vol. 59, no. 3, pp. 506-511, 2010. 


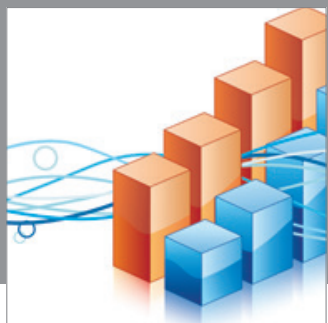

Advances in

Operations Research

mansans

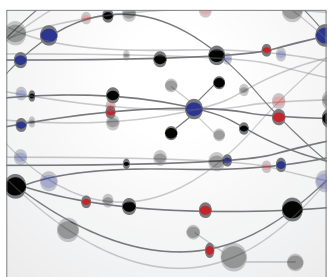

The Scientific World Journal
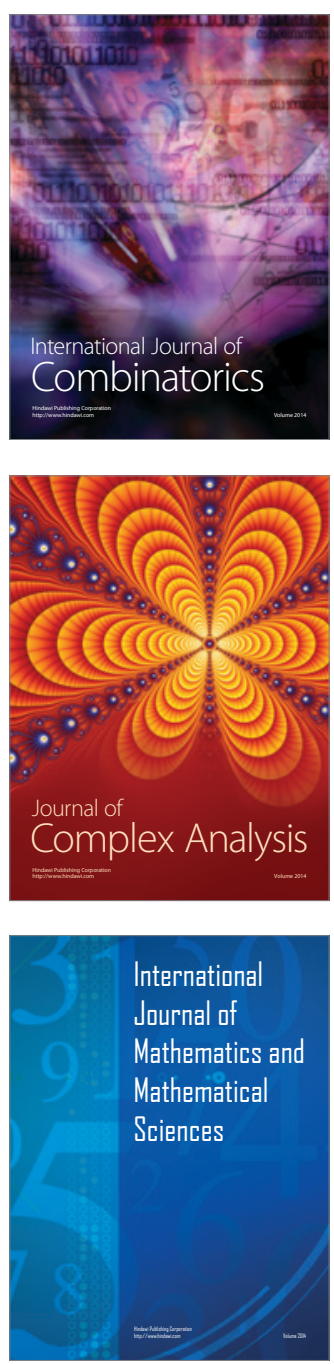
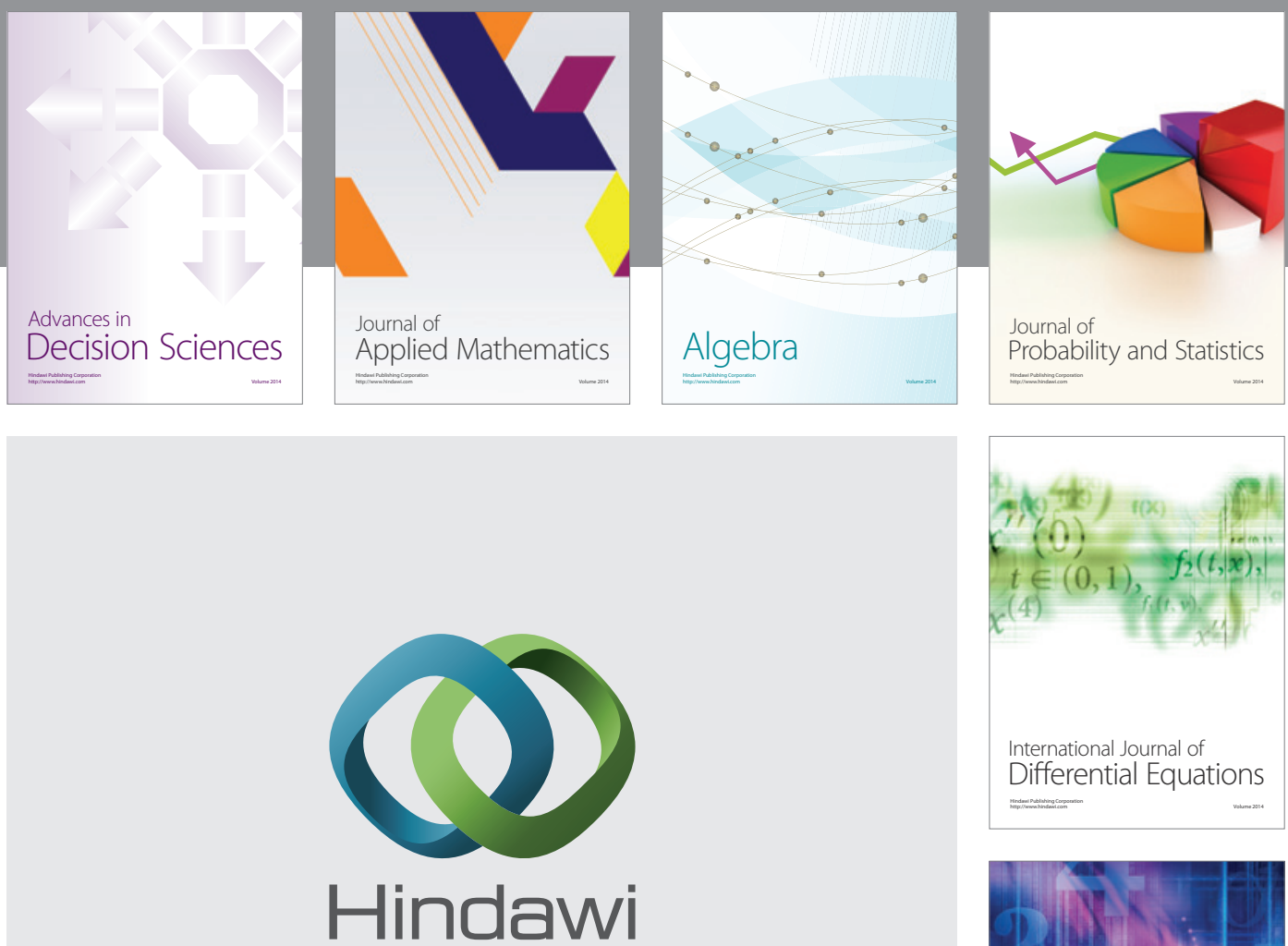

Submit your manuscripts at http://www.hindawi.com
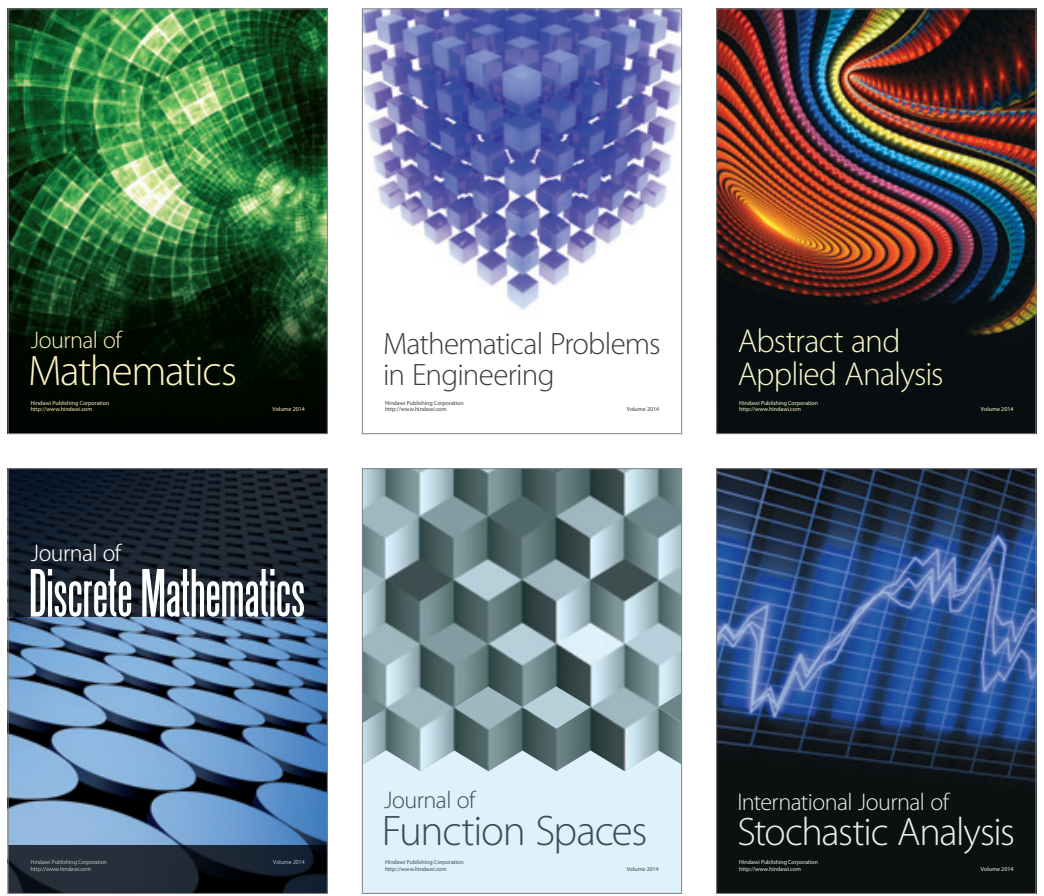

Journal of

Function Spaces

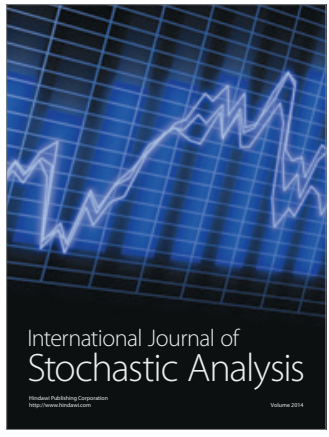

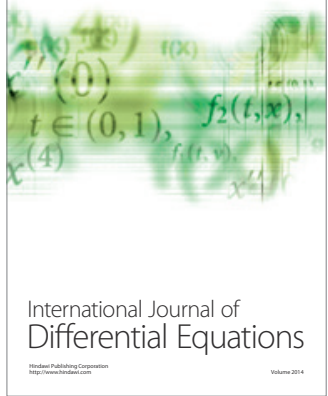
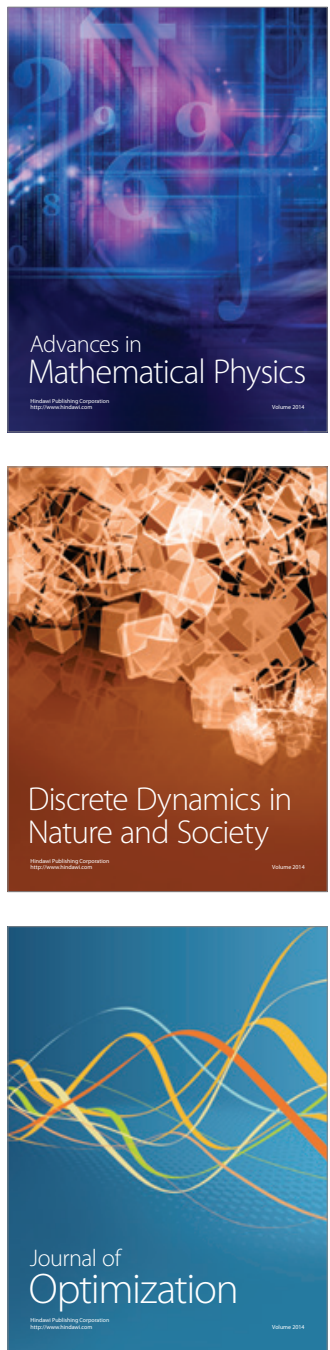